\title{
Deexcitation Dynamics of Superhydrogenated Polycyclic Aromatic Hydrocarbon Cations after Soft-x-Ray Absorption
}

\author{
G. Reitsma, ${ }^{1, *}$ L. Boschman, ${ }^{1,2}$ M. J. Deuzeman, ${ }^{1}$ O. González-Magaña, ${ }^{1}$ S. Hoekstra, ${ }^{3}$ S. Cazaux, ${ }^{2}$ \\ R. Hoekstra, ${ }^{1}$ and T. Schlathölter ${ }^{1}$ \\ ${ }^{1}$ Zernike Institute for Advanced Materials, University of Groningen, Nijenborgh 4, 9747AG Groningen, The Netherlands \\ ${ }^{2}$ Kapteyn Astronomical Institute, University of Groningen, P.O. Box 800, 9700AV Groningen, The Netherlands \\ ${ }^{3}$ Van Swinderen Institute, University of Groningen, Nijenborgh 4, 9747AG Groningen, The Netherlands
}

(Received 7 April 2014; published 30 July 2014)

\begin{abstract}
We have investigated the response of superhydrogenated gas-phase coronene cations upon soft x-ray absorption. Carbon $(1 \mathrm{~s}) \longrightarrow \pi^{\star}$ transitions were resonantly excited at $h \nu=285 \mathrm{eV}$. The resulting core hole is then filled in an Auger decay process, with the excess energy being released in the form of an Auger electron. Predominantly highly excited dications are thus formed, which cool down by hydrogen emission. In superhydrogenated systems, the additional $\mathrm{H}$ atoms act as a buffer, quenching loss of native $\mathrm{H}$ atoms and molecular fragmentation. Dissociation and transition state energies for several $\mathrm{H}$ loss channels were computed by means of density functional theory. Using these energies as input into an Arrhenius-type cascade model, very good agreement with the experimental data is found. The results have important implications for the survival of polyaromatic hydrocarbons in the interstellar medium and reflect key aspects of graphene hydrogenation.
\end{abstract}

DOI: 10.1103/PhysRevLett.113.053002

PACS numbers: 32.80.Aa, 33.80.-b, 95.30.Ft

Hydrogenation of polycyclic aromatic hydrocarbons (PAHs) of all sizes, ranging from graphene layers to small PAH molecules, leads to strong electronic and structural changes, which are relevant from both an applied and a fundamental point of view. The adsorption of hydrogen changes the state of the functionalized $\mathrm{C}$ atom from $s p^{2}$ to $s p^{3}$ and removes $\pi$ electrons from the valence shells. Hydrogenation opens up the graphene band gap in a tunable fashion [1], which is a requirement for using graphene's excellent conductive properties in electronic circuits. Interestingly, the modification of the graphene band gap induced by hydrogenation is reversible, whereas the accompanying structural changes appear to be permanent [2]. Furthermore, graphene related materials currently are studied as promising physisorption substrates for molecular hydrogen storage [3], a prerequisite for "clean" hydrogen powered transport. Reversible hydrogenation has already been successfully applied to, e.g., single walled carbon nanotubes [4], but only layered graphene-based structures with tunable spacing seem to promise sufficient storage capacity between the graphene sheets [3,5]. In order to study the electronic and structural effects on the atomic level in an isolated system, PAH molecules are excellent test bed candidates.

On itself, hydrogenation of PAHs attracts great astrophysical interest because of the yet unexplained high abundance of molecular hydrogen in many astrophysical environments which might be explained by cycles of successive atomic hydrogenation-leading to superhydrogenated PAHs - followed by $\mathrm{H}_{2}$ abstraction [6,7]. Furthermore, in the harsh conditions of the interstellar medium, PAH formation may well be outcompeted by PAH destruction [8]. Laboratory studies on the stability of PAHs to photonic $[9,10]$ and ionic interactions [11-13] indicate, namely, that the PAH lifetimes are substantially shorter than all the current model PAH formation rates [14]. However, hydrogenation is likely to fundamentally alter the PAH response upon absorption of energetic photons.

In this Letter, we report on the photoexcitation of superhydrogenated PAHs and their subsequent deexcitation pathways. The experimental data are interpreted by means of a cascade model, in which theoretical dissociation energies are used to relate molecular excitation to experimentally observed branching ratios. The binding energies of the different hydrogenation stages were calculated by density functional theory (DFT). These energies allow us to relate the level of superhydrogenation to changes in $\mathrm{H}$ loss and geometry of the PAH. The combination of soft x-ray photoionization of superhydrogenated gas-phase PAH cations with quantum chemical calculations is a powerful approach to advance the understanding of the structural and dynamical effects of graphene and PAH hydrogenation. Soft x-ray absorption near the carbon $K$ edge leaves an inner shell vacancy, which gets filled in an Auger decay process. The molecular excitation results predominantly in $\mathrm{H}$ loss, either from the dication (for photoexcitation) or from the trication (for photoionization). In Fig. 1(a) the photoionization and the photoexcitation processes and the subsequent Auger process are displayed schematically.

The experiments were carried out using our tandem mass-spectrometer apparatus $[15,16]$, which was interfaced with the U49/2 PGM1 soft $x$-ray beam line at the BESSY II 


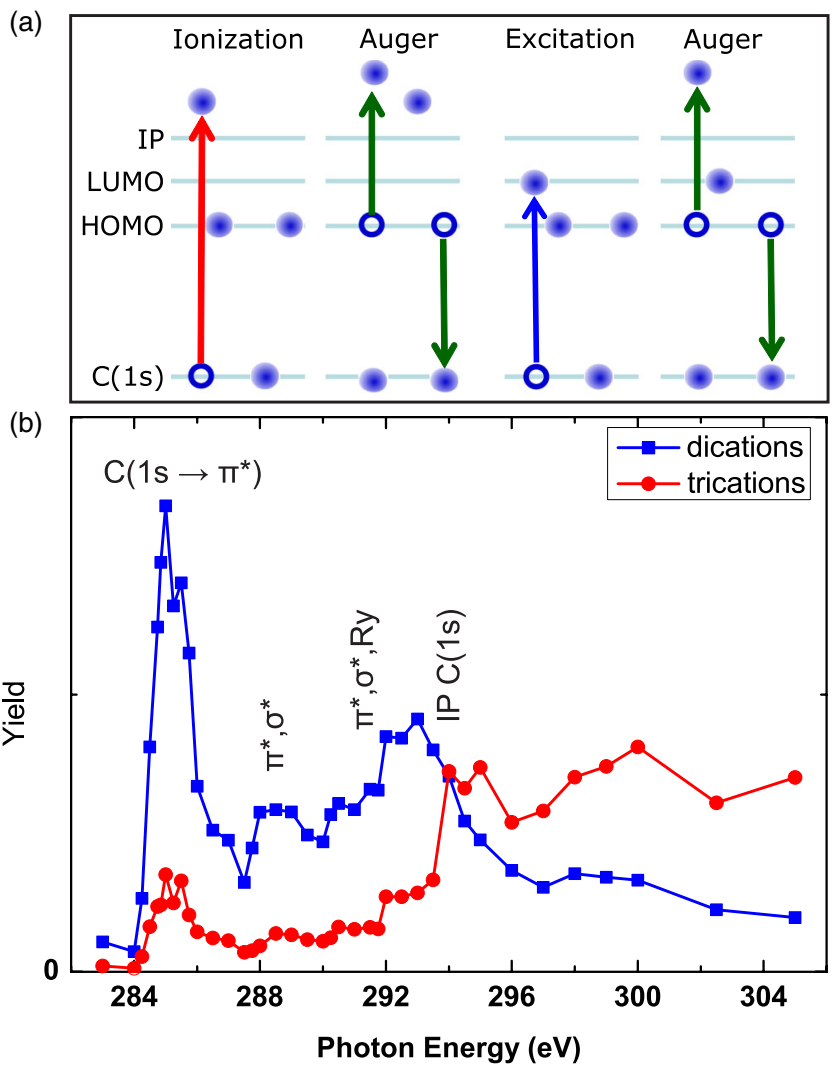

FIG. 1 (color online). (a) A schematic representation of the relevant processes: photoionization (left) and photoexcitation (right). In both cases the inner shell vacancy gets filled by an auger decay. (b) NEXAMS scans of the dication (blue) and trication (red) yield at $m=292-300$ amu after soft X-ray absorption of coronene cations, normalized to initial trap content, photon flux, and irradiation time. As the target is cationic, photoionization (red) mainly leads to tricationic and photoexcitation (blue) to dicationic products.

synchrotron facility at Helmholtz Zentrum Berlin. Nearedge x-ray absorption mass spectrometry (NEXAMS) has been introduced recently as a new tool to study fragmentation pathways of peptides and proteins $[17,18]$. In the present experiments, unhydrogenated gas phase coronene cations $\left(\mathrm{C}_{24} \mathrm{H}_{12}^{+}, M / q=300\right)$ were produced by means of electrospray ionization, mass selected by a radiofrequency (RF) mass filter and trapped in a RF ion trap. The (super) hydrogenation of the coronene (cor) cations was achieved by exposure to an atomic hydrogen beam produced by a $23 \mathrm{MHz}$ Slevin-type hydrogen discharge source $[19,20]$. By variation of the $\mathrm{H}$ exposure times, the degree of hydrogenation was varied over a wide range. Single hydrogenation of a coronene radical cation is followed by pairwise adsorption of $\mathrm{H}$ atoms, giving rise to $[\text { cor }+(2 n+1) \mathrm{H}]^{+}$ $(n=0,1,2,3, \ldots)$ cations, analogous to the fact that $\mathrm{H}$ adsorption on graphene favors $\mathrm{H}$ dimer formation [21].

The trapped cations were exposed to soft x-rays around the $\mathrm{C} K$ edge. Typical photon fluxes varied between $3 \times 10^{12}$ and $1.5 \times 10^{13} \mathrm{~s}^{-1}$ at an energy resolution of
$\Delta E=150 \mathrm{meV}$. The exposure time was varied between 1.0 and $1.8 \mathrm{~s}$. Time-of-flight mass spectrometry of the extracted trap content was then employed to analyze the photoionization products. From the mass spectra, partial ion yields for the various cationic fragments were determined. The ion yields were then normalized to the photon flux, the photon irradiation time, and the initial trap content, to obtain reproducible relative cross sections.

A strong peak in all the mass spectra for nonhydrogenated and superhydrogenated coronene cations, is due to the intact coronene dication and the associated dications which lost $2,4,6$, and 8 hydrogen atoms $((M / q)=150$, $149,148,147$, and 146, $\left.[\operatorname{cor}-m(2 \mathrm{H})]^{2+}(m=0,1,2,3, \ldots)\right)$. For the pure $\mathrm{C}_{24} \mathrm{H}_{12}^{+}$precursor ion, Fig. 1(b) (blue spectrum) displays the summed yields of all these dications as a function of photon energy (i.e., a NEXAMS scan). The red curve shows a NEXAMS scan in which the intensities of the tricationic features, $[\mathrm{cor}-\mathrm{m}(2 \mathrm{H})]^{3+}$, are integrated. The dication yield (blue spectrum) exhibits a sharp resonance at $285 \mathrm{eV}$, which is due to a $\mathrm{C}(1 s) \longrightarrow \pi^{*}$ transition of the coronene cations. The $K$ shell vacancy is filled by an Auger ionization process, predominantly leading to the production of electronically excited coronene dications [Fig. 1(a)]. At higher photon energies other weaker transitions into bound and unbound states occur. To illustrate, discuss, and interpret the effect of superhydrogenation on ionization and fragmentation of PAHs we focus on the dominant photoabsorption channel, namely, the $\mathrm{C}(1 s) \longrightarrow \pi^{*}$ excitation leading to electronically excited dicationic PAHs, which are subsequently transformed into vibrationally hot species by means of intramolecular vibrational-energy redistribution.

Figure 2 displays four zooms of the dication spectral range in the mass spectra after $285 \mathrm{eV}$ photoabsorption for coronene in different degrees of hydrogenation (black spectra). For comparison, the red spectra show the initial monocation mass spectra prior to photoabsorption. Figure 2(a) shows the reference spectrum of pristine $\mathrm{cor}^{+}$. The intact coronene peak $(m=0)$ has a comparable intensity as that of the $2 \times 2 \mathrm{H}$ loss peak $(m=2)$. $2 \mathrm{H}$ loss $(m=1)$ is clearly strongest. More than $68 \%$ of the $[\text { cor }-(2 m) \mathrm{H}]^{2+}$ ions were subject to $\mathrm{H}$ loss $(m>0)$. For Fig. 2(b), the target was exposed to $\mathrm{H}$ atoms for $0.2 \mathrm{~s}$, which primarily leads to single coronene hydrogenation $\left([\text { cor }+\mathrm{H}]^{+}\right)$. Attachment of a single $\mathrm{H}$ atom dramatically alters the effect of photoabsorption: the intact coronene dication $(m=0)$ now dominates over the $\mathrm{H}$ loss channels $(m>0)$. Figures 2(c) and 2(d) show mass spectra obtained after longer $\mathrm{H}$ exposure times. For Fig. 2(c), $6 \mathrm{~s}$ of exposure led to predominantly $n=1$ and $n=2$. The dissociation channels $m=1$ and $m=2$ are clearly weaker and the peaks due to intact coronene dications with still 1 or 3 additional hydrogen atoms attached are clearly visible. In Fig. 2(d) the hydrogenation is pushed to even higher degrees of superhydrogenation. The experiments clearly show that 


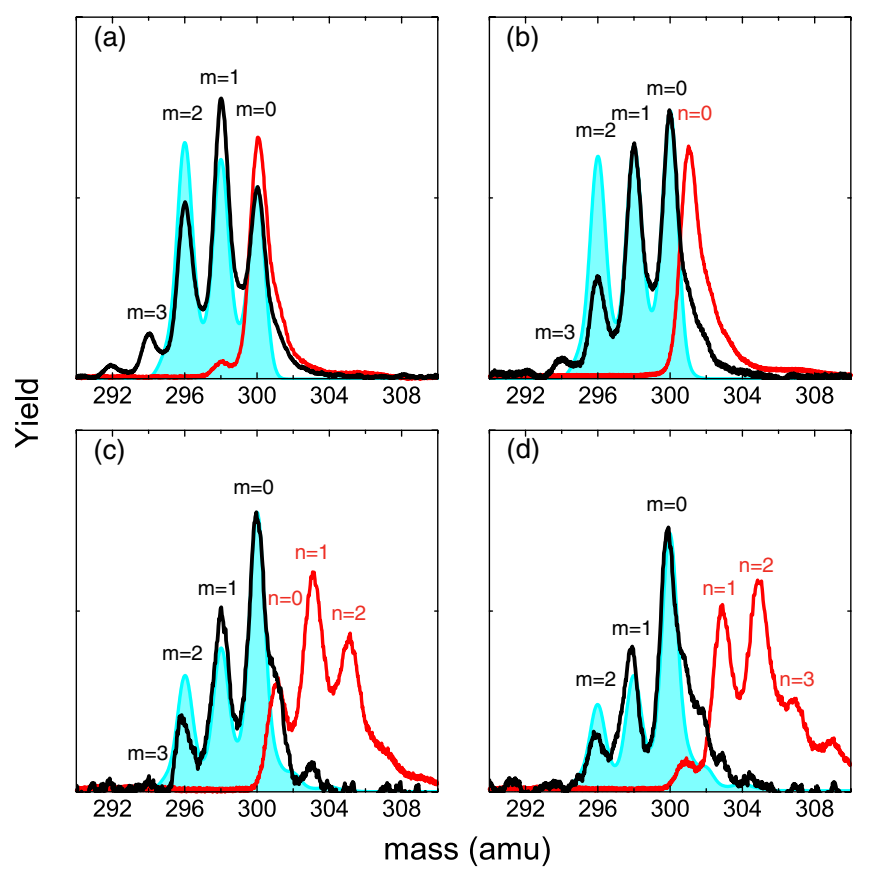

FIG. 2 (color online). Mass spectra of (superhydrogenated) coronene after irradiation with $285 \mathrm{eV}$ photons (black). The mass spectra in red represent the initial hydrogenation state distribution of the target cations. The cyan spectra show the corresponding model fragmentation spectra. Details about the model and the labels, $m$ and $n$, are given in the text. The model spectra are normalized to the $M=300$ peak in the black fragmentation spectrum. The red reference spectra are not normalized.

superhydrogenation acts as a buffer and thus increases PAH stability. The strongly alternating peak intensities, i.e., the strong dominance of even over odd numbers of losses, hints at dissociation by sequential $\mathrm{H}$ loss with alternating dissociation energies. Such deexcitation processes are thought to be of statistical nature, occurring after intramolecular vibrational redistribution of internal energy.

Under the assumption that in vibrationally hot superhydrogenated PAH dications, consecutive $\mathrm{H}$ losses compete with infrared (IR) photon emission, one may use a basic cascade model to describe the relaxation of the PAH molecules. The main ingredients for such a model are the infrared photon emission rate, the hydrogen binding energy, and the internal energy. In this scenario, the dissociation probability for dissociation step $i$ can be written as

$$
P_{i}^{\mathrm{diss}}=\frac{k_{i} e^{-E_{i} / k T_{i}^{\mathrm{av}}}}{\frac{k_{\mathrm{IR}}}{n_{\max }+1}+k_{i} e^{-E_{i} / k T_{i}^{\mathrm{av}}}},
$$

with preexponential factor $k_{i}=\left(k T_{i}^{\text {eff }} / h\right) \exp (1+(\Delta S / R))$ and average PAH temperature $T_{i}^{\mathrm{av}}=\sqrt{T_{i, 0}^{\mathrm{eff}} T_{i, n_{\max }}^{\mathrm{eff}}} \cdot T_{i, 0}^{\mathrm{eff}}$ and $T_{i, n_{\max }}^{\text {eff }}$ correspond to the effective temperatures when 0 or $n_{\max }$ infrared photons have been emitted, respectively [14]. The effective temperature at step $i$ is defined as

$$
T_{i}^{\mathrm{eff}}=2000\left(\frac{T_{E, i}}{N_{C}}\right)^{0.4}\left(1-0.2 \frac{E_{i}}{T_{E, i}}\right)
$$

in which $E_{i}$ is the dissociation energy and $T_{E, i}$ is the total vibrational energy at step $i$ [14]. We further set the maximum number of emitted photons $n_{\max }=6$ [14] and the entropy change $\Delta S=2.5 \mathrm{cal} / \mathrm{mol}$ [22]. For coronene the number of $\mathrm{C}$ atoms is $N_{C}=24$. Typical IR emission rates are between 1 and $1000 \mathrm{~s}^{-1}$ [9]. We adopted $k_{\mathrm{IR}}=10 \mathrm{~s}^{-1}$ from Ref. [23]. $R, h$, and $k$ are the gas constant, Planck's constant, and the Boltzmann constant, respectively. We used DFT to determine $E_{i}$ for atomic and molecular hydrogen loss and the transition states for molecular hydrogen loss. The calculations were performed by using the B3LYP [24,25] functional and a 6-31G(d) basis set as implemented in the GAUSSIAN09 package [26]. The molecular structures were optimized to a minimum (all real frequencies) or to a transition state (one imaginary frequency) after which the frequencies were calculated. The energies were obtained by subtracting the zero-point energy (corrected by the empirical scaling factor 0.9806 [27]) from the total energy. For the calculations of the hydrogen binding energies to the superhydrogenated PAHs, we used the reaction routes from Ref. [6]. Figure 3 displays an overview of the results. Analogous to the hydrogen adsorption induced structural changes in graphene [2], the inset in Fig. 3 demonstrates the dramatic structural changes of coronene, induced by the addition of, e.g., $7 \mathrm{H}$ atoms $(n=3)$. This implies: (i) large PAHs can be viewed as model systems for studying graphene properties in the gas phase; and (ii) hydrogenation induced structural changes break the molecular symmetry,

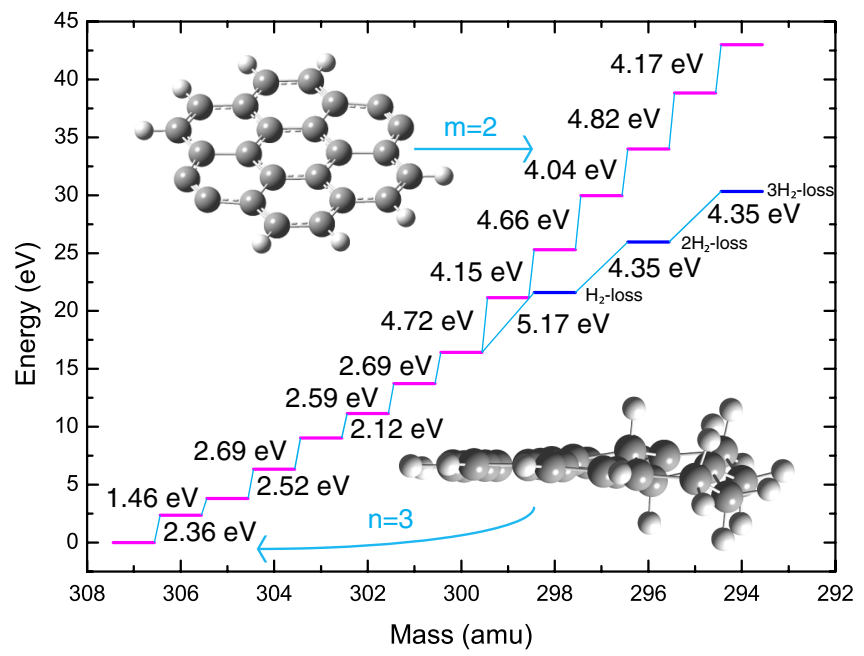

FIG. 3 (color online). Overview of dissociation energies of $\mathrm{H}$ atoms, relative to the total energy of coronene $+4 \mathrm{H}$. The levels in purple are the dissociation energies of single $\mathrm{H}$ atoms and the blue levels are the barriers for emission of $\mathrm{H}_{2}$. The displayed molecular structures of $m=2$ (top) and $n=3$ (bottom) clearly show breaking of planar symmetry after superhydrogenation. 
certainly leading to an altered infrared emission spectrum that could potentially be used for identification of these species.

The branching ratios for the loss channel corresponding to the $i$ th hydrogen loss $A_{i}$ can then be determined using

$$
A_{i}=\int\left[1-P_{i+1}^{\mathrm{diss}}\left(T_{E}\right)\right] \prod_{k=0}^{i} P_{k}^{\mathrm{diss}}\left(T_{E}\right) P\left(T_{E}\right) d T_{E} .
$$

$P\left(T_{E}\right)$ is the normalized vibrational energy distribution of the system. Under the assumption that intramolecular vibrational energy redistribution quickly transforms electronic excitation into vibrational energy, it is appropriate to extract an approximation of $P\left(T_{E}\right)$ from an Auger spectrum. We assumed that the electronic orbital structure is similar for aromatic hydrocarbons and therefore used an Auger spectrum of benzene (Ref. [28], Fig. 7). To obtain the excitation energy distribution $3.7 \mathrm{eV}$ was added to account for the additional excitation energy which was deposited by the $\mathrm{C}(1 s) \longrightarrow \pi^{*}$ transition [Fig. 1(a)]. The cyan spectra in Fig. 2 represent the predicted fragmentation mass spectra, obtained by starting out from the initial hydrogenation state distribution (red). Note, that the calculations were restricted to superhydrogenation of up to 7 atoms and to loss of up to $6 \mathrm{H}$ atoms from bare coronene. The peak for $m=2$ is clearly overestimated by the model, which can be explained by the fact that the associated high vibrational energies opens up channels which were not included in the model. However, good agreement with the experimental data is evident and allows for drawing the following important conclusions. (i) The quenching of odd numbered H loss channels $(M=295,297,299)$ is reproduced in the calculation and originates from the alternating $\mathrm{H}$ binding energies $(4.72,4.15,4.66$, and $4.04 \mathrm{eV}$ for the first, second, third, and fourth $\mathrm{H}$ loss, respectively). Single $\mathrm{H}$ loss is found to outcompete $\mathrm{H}_{2}$ loss, which is associated with a $5.2 \mathrm{eV}$ barrier. (ii) For the bare coronene molecule [Fig. 2(a)] experiment and model both show, that emission of $2 \mathrm{H}$ and $4 \mathrm{H}$ dominates over the nondissociative channel. Addition of a single $\mathrm{H}$ atom dramatically shifts the distribution with a slight overestimation of the loss channels by the model [Fig. 2(b)]. Additional $\mathrm{H}$ atoms are boiled off very efficiently because of their much lower binding energies of 2.7, 2.6, 2.1, 2.7, 2.5, 1.5, and $2.4 \mathrm{eV}$ for the first $7 \mathrm{H}$ atoms. The model confirms that most of the energy is released during the evaporation of these additional $\mathrm{H}$ atoms, causing an increase in the survival of intact molecules.

In this Letter, we report on the first experimental study of the dissociation of superhydrogenated PAHs. We compared the experiments with a cascade model that allowed us to reproduce the mass spectra with a high accuracy. Both the experiments and the model show a strong increase in the population of the intact molecule and a decrease in the population of the molecular fragments. The combination of experimental data and a rather simple model indicates: (i) Superhydrogenation of PAHs implies removal of bonding $\pi$ electrons and leads to local puckering of the graphenelike planar honeycomb structure. Not only are the binding energies of the additional hydrogen atoms relatively low $(\approx 2.5 \mathrm{eV})$, but as a consequence of superhydrogenation, also the $\mathrm{C}$ backbone is locally weakened. Despite this weakening, superhydrogenation clearly acts as a protection mechanism for PAH molecules. (ii) Superhydrogenation may explain the existence of large amounts of PAHs in the interstellar medium, as deexcitation by $\mathrm{H}$ loss protects the $\mathrm{C}$ backbone from fragmentation.

We gratefully acknowledge financial support by the Nederlandse organisatie voor Wetenschappelijk Onderzoek (NWO) within the Dutch Astrochemistry Network (DAN). We thank the Helmholtz Zentrum Berlin for allocation of synchrotron beamtime and acknowledge funding from the European Community's Seventh Framework Programme (FP7/2007-2013, Grant No. 312284).

*.reitsma@rug.nl

[1] D. C. Elias et al., Science 323, 610 (2009).

[2] A. Castellanos-Gomez, M. Wojtaszek, Arramel, N. Tombros, and B. J. van Wees, Small 8, 1607 (2012).

[3] M. Pumera, Energy Environ. Sci. 4, 668 (2011).

[4] A. Nikitin, H. Ogasawara, D. Mann, R. Denecke, Z. Zhang, H. Dai, K. Cho, and A. Nilsson, Phys. Rev. Lett. 95, 225507 (2005).

[5] S. Patchkovskii, J. S. Tse, S. N. Yurchenko, L. Zhechkov, T. Heine, and G. Seifert, Proc. Natl. Acad. Sci. U.S.A. 102, 10439 (2005).

[6] E. Rauls and L. Hornekær, Astrophys. J. 679, 531 (2008).

[7] J. D. Thrower, B. Jørgensen, E. E. Friis, S. Baouche, V. Mennella, A. C. Luntz, M. Andersen, B. Hammer, and L. Hornekær, Astrophys. J. 752, 3 (2012).

[8] A. G. G. M. Tielens, Rev. Mod. Phys. 85, 1021 (2013).

[9] H. W. Jochims, E. Ruhl, H. Baumgartel, S. Tobita, and S. Leach, Astrophys. J. 420, 307 (1994).

[10] S. Leach, J. H. D. Eland, and S. Price, J. Phys. Chem. 93, 7583 (1989).

[11] A. I. S. Holm et al., Phys. Rev. Lett. 105, 213401 (2010).

[12] G. Reitsma, H. Zettergren, L. Boschman, E. Bodewits, R. Hoekstra, and T. Schlathölter, J. Phys. B 46, 245201 (2013).

[13] G. Reitsma, H. Zettergren, S. Martin, R. Brédy, L. Chen, J. Bernard, R. Hoekstra, and T. Schlathölter, J. Phys. B 45, 215201 (2012).

[14] E. R. Micelotta, A. P. Jones, and A. G. G. M. Tielens, Astron. Astrophys. 510, A37 (2010).

[15] S. Bari, R. Hoekstra, and T. Schlathölter, Phys. Chem. Chem. Phys. 12, 3376 (2010).

[16] O. González-Magaña et al., Phys. Rev. A 87, 032702 (2013).

[17] O. González-Magaña, G. Reitsma, M. Tiemens, L. Boschman, R. Hoekstra, and T. Schlathölter, J. Phys. Chem. A 116, 10745 (2012). 
[18] A. R. Milosavljević, F. Canon, C. Nicolas, C. Miron, L. Nahon, and A. Giuliani, J. Phys. Chem. Lett. 3, 1191 (2012).

[19] L. Boschman, G. Reitsma, S. Cazaux, T. Schlathölter, R. Hoekstra, M. Spaans, and O. González-Magaña, Astrophys. J. Lett. 761, L33 (2012).

[20] R. Hoekstra, A. R. Schlatmann, F. J. de Heer, and R. Morgenstern, J. Phys. B 22, L603 (1989).

[21] R. Balog, B. Jørgensen, J. Wells, E. Lægsgaard, P. Hofmann, F. Besenbacher, and L. Hornekær, J. Am. Chem. Soc. 131, 8744 (2009).
[22] Y. Ling and C. Lifshitz, J. Phys. Chem. A 102, 708 (1998).

[23] R. C. Dunbar, J. H. Chen, H. Y. So, and B. Asamoto, J. Chem. Phys. 86, 2081 (1987).

[24] A. D. Becke, J. Chem. Phys. 98, 5648 (1993).

[25] C. Lee, W. Yang, and R. G. Parr, Phys. Rev. B 37, 785 (1988).

[26] M. J. Frisch et al., Gaussian Inc., Wallingford CT, 2009.

[27] A. P. Scott and L. Radom, J. Phys. Chem. 100, 16502 (1996).

[28] R. R. Rye and J.E. Houston, Acc. Chem. Res. 17, 41 (1984). 